\title{
Prevalence and clinical significance of fetal tricuspid regurgitation during the third trimester of pregnancy
}

Karina Reis de Melo Lopes 1

iD https://orcid.org/0000-0001-7177-145X

Emanuela Virginia Vale Cavalcante 2

(iD) https://orcid.org/0000-0002-9685-5382

Narjara Tiane Lopes de Melo 3

iD https://orcid.org/0000-0002-4602-7503

Susi Araújo Alves 4

iD https://orcid.org/0000-0001-7581-180X

\author{
João Guilherme Bezerra Alves 5 \\ iD https://orcid.org/0000-0002-9170-0808 \\ Alex Sandro Rolland Souza 6 \\ iD https://orcid.org/0000-0001-7039-2052
}

\footnotetext{
1 Fetal Medicine Unit, Biofetoclinical. Praça do Derby, 149. $7^{\circ}$ andar. Derby. CEP: 52.010-140. Recife, PE, Brazil. E-mail: karinarml@gmail.com 2-4 Faculdade Pernambucana de Saúde. Recife, PE, Brazil.

5,6 Post Graduation Department. Instituto de Medicina Integral Prof. Fernando Figueira. Recife, PE, Brazil.
}

\begin{abstract}
Objectives: the aim of this study was to determine the prevalence of fetal tricuspid valve regurgitation (TR) during the third trimester of low-risk pregnancies and to assess its clinical significance on neonates.

Methods: this is a cross-sectional study including 330 singleton fetuses referred for routine fetal echocardiography during 3 rd trimester in a fetal medicine center in Recife, Brazil. The presence and degree of tricuspid regurgitation were analyzed. Whenever TR was identified on fetal echocardiography, postnatal data, including the results of postnatal echocardiography were reviewed.

Results: the prevalence of tricuspid regurgitation was $10.0 \%(n=33)$ in the study population. Regarding regurgitation degree, $90.9 \%(n=30)$ presented mild regurgitation and none presented important TR. Postnatal data was obtained from 21 neonates. Twenty of them were discharged without any complications, and one presented respiratory distress due to prematurity. Transthoracic echocardiography was performed in $66.7 \%(n=14)$ of the neonates and it was normal in $92.9 \%(n=13)$ of them. One neonate, $7.1 \%$, persisted with tricuspid regurgitation, but had no other findings.

Conclusions: tricuspid regurgitation in fetuses with normal cardiac anatomy during the 3 rd trimester is a common condition in low-risk pregnancies, and is not associated with cardiac abnormalities or need for neonatal intervention.

Key words Tricuspid valve insufficiency, Fetal heart, Congenital heart defect, Prenatal diagnosis, Echocardiography doppler
\end{abstract}




\section{Introduction}

Tricuspid valve regurgitation (TR) is a frequent finding on Doppler examination of the heart in newborns, children and adults, with reported prevalence of more than $80 \%$ in individuals with normal hearts. ${ }^{1}$ Differently, in fetuses, the reported prevalence is below $7 \% .2,3$

Fetal TR has long been associated with abnormal fetal physiology. Increased preload or afterload secondary to myocardial impairment, nonimmune hydrops, arrhythmias and related to congenital heart defects (CHD) such as Ebstein's disease, pulmonary atresia with ventricular septal defect and ductal constriction may result in tricuspid regurgitation.3-5 There has been a lot of investigation regarding the potential role of tricuspid regurgitation in the first and second trimesters of pregnancy as a marker for chromosomal abnormalities or cardiac defects. It is well established that the prevalence of fetal TR increases with increased nuchal translucency measurement and in the presence of other cardiac defects, but seems to decrease with progression of gestation. 6-10

A recent metanalysis demonstrated that the detection of TR in the first trimester increases the risk of CHD. However, the strength of association between TR and CHD was greater when considering fetuses at risk for CHD, such as those with increased nuchal translucency, while TR did not show any association with CHD when detected in a population at low risk for cardiac defects. Thus, isolated TR in the first trimester does not seem to be a strong predictor of CHD. ${ }^{7-9}$ Furthermore, recent studies suggest that, in uncomplicated pregnancies of euploid fetuses with normal cardiac anatomy, tricuspid regurgitation might not be associated with other pathological findings. ${ }^{7-10}$

To date, the evidence has been contradictory as to whether fetal TR in the second trimester is predictive of other disorders. Fetuses with TR in the second trimester have been reported to present a greater risk of aneuploidy, cardiac defects and adverse neonatal outcomes in fetuses with intrauterine growth restriction.11,12 On the other hand, some studies suggest that isolated mild tricuspid regurgitation in this stage of pregnancy is benign in fetuses with normal cardiac anatomy and that these fetuses might not have a higher incidence of major cardiac disorders after birth.2,10

Although extensively studied during the first and early second trimesters, little is known about the role of TR in the late second and third trimesters of pregnancy. Some studies suggest that the prevalence of
TR decreases during pregnancy. $6-10$ Others have reported higher prevalence in late pregnancy. 10,13 Limited information is available regarding normal findings of the fetal heart in late pregnancy. Recent data suggest that the prevalence of mild TR would increase with gestational age and would be a result of the physiologic alterations that occur late in the third trimester. 13

The present study aimed to determine the prevalence of TR in low-risk pregnancies in the third trimester of pregnancy and to assess the clinical significance of this finding on neonatal outcome.

\section{Methods}

A cross-sectional study was performed including all low-risk singleton fetuses referred for routine fetal echocardiography during the 3 rd trimester at Biofeto, a fetal medicine center located in Recife, Pernambuco, Brazil.

Initially, data from all fetal echocardiograms performed from August 1st, 2014 to July 31 st 2015 was collected from Biofeto's database. Whenever one of the following was present, the subject was excluded from the analysis: gestational age $<28$ weeks, women aged over 35 years, the presence of maternal and/or fetal chronic disease (arterial hypertension, diabetes mellitus and preeclampsia, for example), fetal chromosomal abnormality and/or congenital malformations, abnormal nuchal translucency, small for gestational age, multiple pregnancies, fetal cardiac disease and positive family history for congenital heart disease.

Data included mean maternal age, mean gestational age at the time of the fetal echocardiogram, presence and degree of TR, associated findings, postnatal outcome, postnatal echocardiogram, and mean gestational age at delivery.

When tricuspid regurgitation was present on fetal echocardiogram, the pregnant women were contacted and invited to answer an electronic questionnaire sent through the Lime Survey software (Survey Services \& Consulting, Hamburg, Germany). In this questionnaire, they were inquired about gestational age at delivery, postnatal outcome and need for interventions, postnatal diagnosis of heart disease and postnatal echocardiogram data when performed.

All 2D- fetal echocardiograms were performed by a single experienced fetal cardiologist, with a Philips@ (Koninklijke Philips NV, USA) HD 11 XE machine, using a $2-5 \mathrm{Mhz}$ abdominal probe. The fetal heart was imaged in multiple planes and measurements were made according to guidelines published 
by the American Society of Echocardiography. ${ }^{14}$ Tricuspid regurgitation was recorded during ventricular contraction in the apical or basal 4-chamber views using color and pulsed Doppler imaging with an angle of insonation from $0^{\circ}$ to $30^{\circ}$.TR was categorized according to the following parameters: length of the jet into the right atrium (RA), the area of the RA covered by the jet, and duration of the jet in the cardiac cycle. TR was considered mild when the length of jet was less than $1 / 3$ of the distance to the opposite atrial wall, RA area covered by the jet was less than $25 \%$ and the duration was protosystolic; moderate when the length of jet was higher than $2 / 3$, but not reaching the opposite wall, RA covered by the jet in $25 \%$ and the duration was proto-mesosystolic; and severe when the jet reached the opposite atrial wall and was holosystolic (Figure 1). Trivial TR, i.e. regurgitant jets seen on color Doppler mapping that could not be appreciated on pulsed wave Doppler, were not considered for this study. During the study period, patients were usually offered a follow-up scan when a TR was identified.

Sample size was calculated at 97 participants considering a margin of error of $5 \%$, a confidence level of $95 \%$ and an estimated prevalence of tricuspid regurgitation of $6.8 \%, 3$ using Epi info ${ }^{\mathrm{TM}}$ 7.2.2.6 software (Centers for Disease Control and Prevention - CDC, Atlanta, GA, USA).

All data processing was performed using Epi info $^{\text {TM }}$ 7.2.2.6 software (Centers for Disease Control and Prevention - CDC, Atlanta, GA, USA) and numeric data were presented as mean \pm standard deviation (SD) and frequency.

This study was approved by the Research Ethics Committee of Faculdade Pernambucana de Saúde (Pernambuco Faculty of Health) under CAAE number 49200615.8.0000.5569. Signed participant consent was obtained when participants were contacted to complete the questionnaire.

\section{Results}

In the period of study, 6352-D fetal echocardiograms were performed, 330 third trimester low-risk pregnancies were identified according to the eligibility criterion and were included in the study. From the total of 330 pregnancies, 33 fetuses had TR, indicating a prevalence of $10.0 \%$. For postnatal analysis, data from 12 participants could not be obtained. Three of them for contact failure and nine who did not answer the questionnaire (Figure 2).

The mean maternal age of the 33 included cases was 27.2 years old $(\mathrm{SD} \pm 4.1)$ and the mean gestational age at which TR was identified on fetal echocardiogram was 32 weeks $(\mathrm{SD} \pm 3.6)$.

With respect to TR degree of severity, $90.9 \%$ $(\mathrm{n}=30)$ presented a mild regurgitation and $9.1 \%$ $(\mathrm{n}=3)$ were classified as moderate regurgitation. Other findings associated with TR during fetal echocardiography were found in $18.2 \%(n=6)$ of the patients. Four fetuses presented increased ductus arteriosus peak systolic velocity $(>140 \mathrm{~cm} / \mathrm{s})$ not fulfilling arterial duct constriction criteria, though. During follow-up, one fetus had mild pericardial effusion and one mild pleural effusion. These were not related to congestive heart failure and were considered minor findings, resolving spontaneously during pregnancy (Table 1).

From the 33 cases of TR, postnatal data could be obtained in $21(63.6 \%)$. The mean gestational age at delivery was 38.3 weeks $(\mathrm{SD} \pm 1.23)$. At postnatal evaluation, all neonates were discharged from maternity without concerns and were asymptomatic, with exception of one newborn who needed advanced life support due to respiratory distress, secondary to prematurity, a consequence of preterm labor (Table 1).

Among the patients who answered the questionnaire, $66.7 \%(\mathrm{n}=14)$ underwent post-natal echocardiography in the neonatal period, which was normal in 13 neonates. In one case (7.1\%) TR persisted, but there were no other associated findings (Table 1).

\section{Discussion}

Fetal TR was present in $10.0 \%$ of low-risk pregnancies who performed 2-D fetal echocardiography during the $3^{\text {rd }}$ trimester of gestation. Most of them presented mild regurgitation with no other associated conditions, supporting the idea that tricuspid regurgitation might be a physiological finding in this population.

Postnatal echocardiograms were normal in almost all newborns (92.9\%) who presented fetal TR and had an echocardiogram performed after birth. Only one child had persistent TR after birth, but without clinical significance. All the newborns but one, who presented complications related to prematurity, were discharged from maternity without concerns. These data together corroborate the innocuous aspect of fetal TR.

When considering a population of high-risk pregnancies, the association of fetal TR with pathological conditions leading to preload increase, postload increase, myocardial damage, or arrhythmias is more evident, as reported by Respondek et al. 3 In that manner, in our sample, 4 cases presented altered ductus arteriosus flow characterized by increased 
peak systolic velocity, without criteria of ductal constriction. In these cases, tricuspid regurgitation can be explained by the increase of post-load to the right ventricle.

Overall, tricuspid regurgitation was initially strongly associated with pathological status such as chromosomal and cardiac abnormalities, based on studies performed mainly in the first and early second trimesters. However, recent data suggest that TR might have a different meaning. Zhou et al. ${ }^{2}$ evaluated 1429 fetuses between 18-24 weeks of gestation and described a prevalence of mild TR of $6.7 \%$. They concluded that fetuses with isolated mild $\mathrm{TR}$ in the second trimester did not have higher incidence of major cardiac disorders after birth, but had a higher prevalence of minor postnatal congenital cardiac disorders. ${ }^{2}$

Messing et al. 10 investigated the prevalence of fetal TR in 157 low-risk pregnant women at the beginning and middle of the second trimester and postnatal period. At the first moment, 131 fetuses $(83.4 \%)$ presented TR and at the second moment, only 39 fetuses $(24.8 \%)$, suggesting that the prevalence of TR would decrease along with gestational age. At postnatal evaluation, eight children persisted with TR. 10 This high prevalence of TR differs from that obtained by this study and by Gembruch and Smrcek4 and can be justified by the use of three- dimensional (3D) echocardiography associated with spatio-temporal image correlation (STIC). One of the main concerns regarding this technology are the artifacts resulting from fetal movement and maternal respiratory movements, which present with a gain greater than the appropriate and can simulate tricuspid regurgitation. 15 Two-dimensional ultrasonography, used in this study, is the technique of choice for the prenatal diagnosis of congenital heart diseases. It allows accurate description of the intracardiac anatomy, sequential analysis of cardiac structures, and finally the recognition of malformations, cardiac rhythm disorders and function in the prenatal period. 14

Conversely, recent data point out that TR is more frequent later in pregnancy and that in this scenario, it is mainly a physiological finding. For example, Gembruch and Smrcek 4 evaluating 289 second trimester fetuses, estimated a prevalence of TR of $6.23 \%$. In the postnatal echocardiographic evaluation, none of the children presented cardiac alterations. 4 Their results are very similar to those obtained by the present study. In addition, Tague et al. 13 studied 40 third trimester fetuses with structurally normal hearts and reported the very high prevalence of trivial and mild tricuspid regurgitation of $80 \%$. The high prevalence of TR reported in this study, different from ours, can be explained by their

\section{Figure 1}

A) Sonogram of a fetus with normal heart anatomy and mild tricuspid regurgitation at 31.2 weeks' gestation. The image shows reversed flow (arrow) across the tricuspid valve at the level of the 4-chamber view. B) Pulsed Doppler interrogation of the tricuspid valve demonstrating normal forward flow and protosystolic low velocity reverse flow.

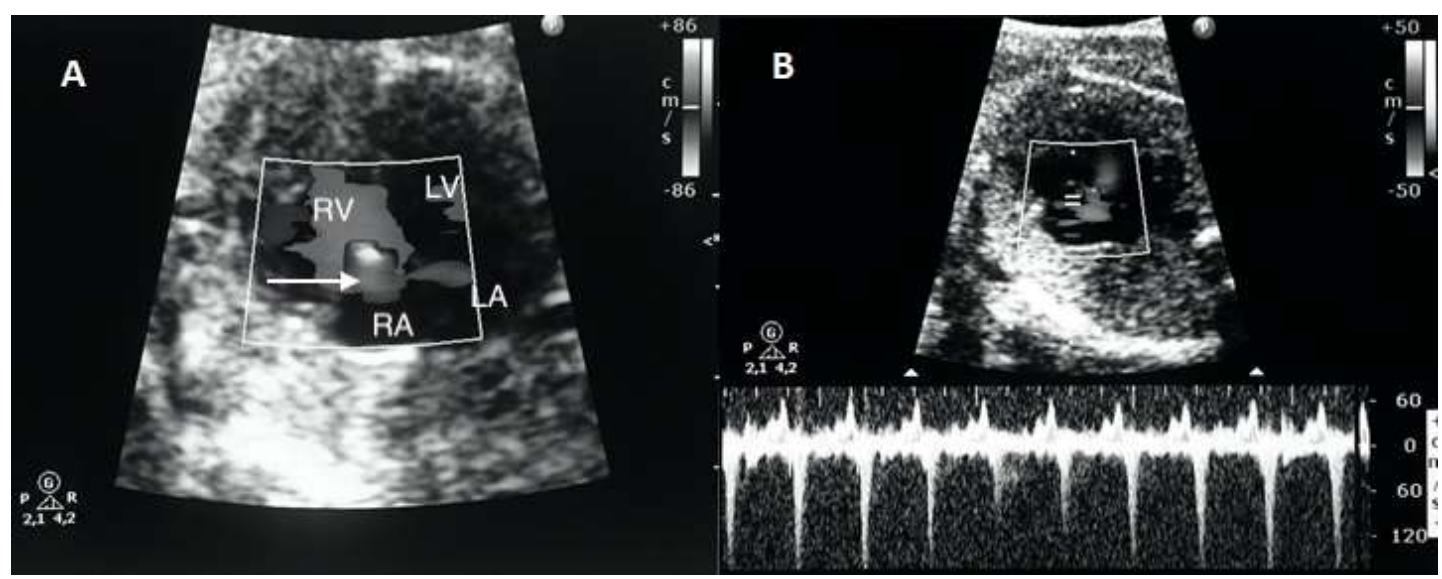

$\mathrm{LA}=$ left atrium; $\mathrm{LV}=$ left ventricle; $\mathrm{RA}=$ right atrium; $\mathrm{RV}=$ right ventricle. 
Figure 2

Sample flow chart.

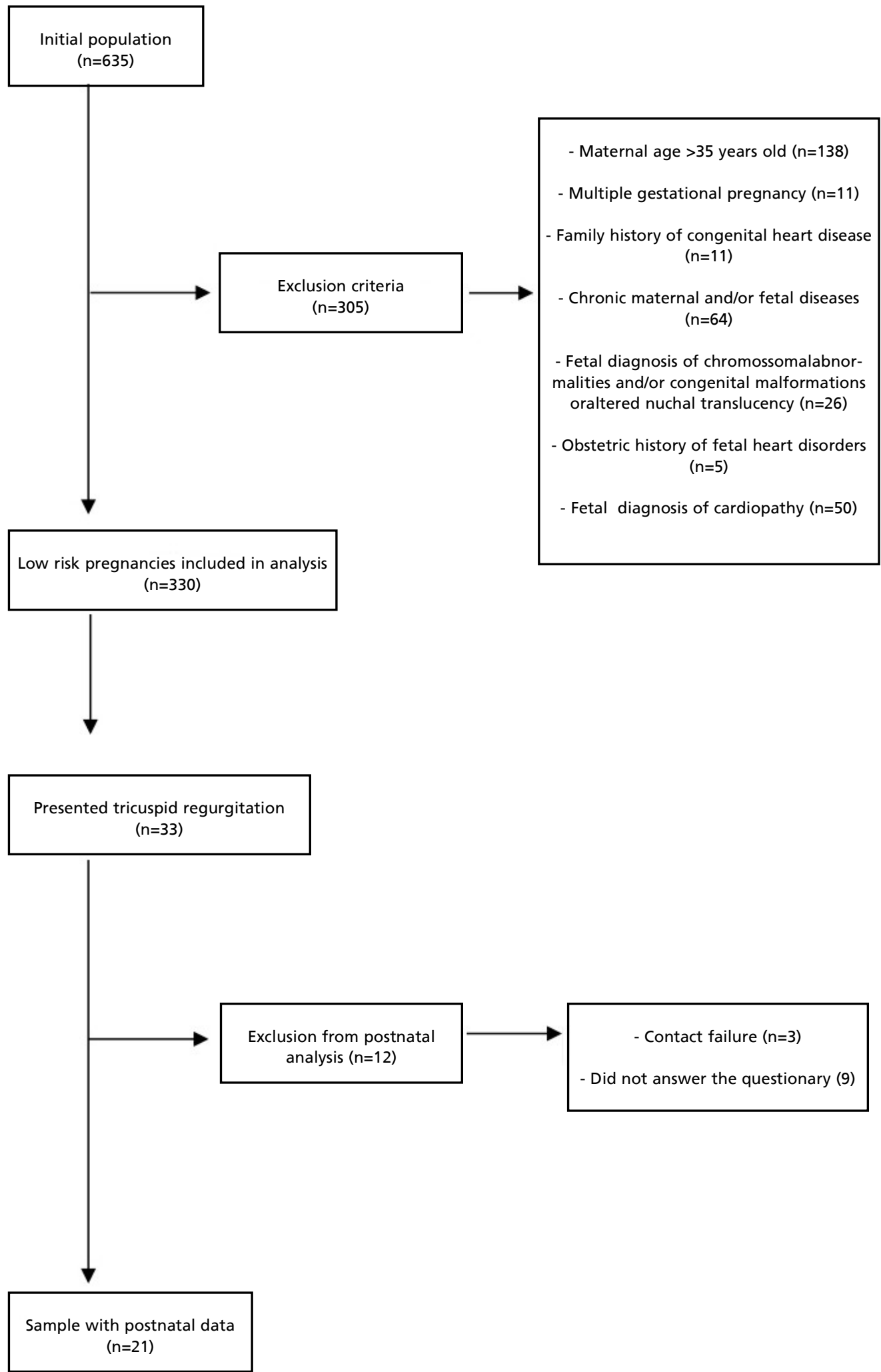




\begin{tabular}{lccc}
\hline \multicolumn{2}{l}{ Perinatal findings and outcome of fetal tricuspid regurgitation. } & & \\
\hline & $\mathbf{N}$ & $\mathbf{n}$ & $\%$ \\
\hline Degree of TR & 33 & 30 & 90.9 \\
$\quad$ Mild & & 3 & 9.1 \\
$\quad$ Moderate & 33 & 4 & 12.1 \\
Associated findings & & 2 & 6.1 \\
$\quad$ Increased arterial duct peak systolic velocity & & & 95.2 \\
$\quad$ Effusions (pericardial or pleural) & 21 & 20 & 4.8 \\
Postnatal outcome & & 1 & 92.9 \\
$\quad$ Uneventful & 14 & 13 & 7.1 \\
$\quad$ Respiratory support due to prematurity & & 1 & \\
Postnatal echocardiogram & & & \\
$\quad$ Normal & & & \\
Persistent TR & & & \\
&
\end{tabular}

$\mathrm{TR}=$ Tricuspid regurgitation .

inclusion of trivial TR cases, defined as the regurgitations that can only be detected by color Doppler investigation, but not by pulsed Doppler investigation. Another possible explanation for such a high prevalence of TR would be its population's gestational age (34 to 38 weeks), corroborating the idea that TR prevalence increases with gestational age. They also reported right/left disproportion of cardiac chambers and an increase in aortic isthmus and arterial duct diastolic flow suggesting that $T R$ is possibly related to the physiological hemodynamic changes of late pregnancy. ${ }^{13}$ We did not include trivial TR in our study due to its obvious physiological role. Our aim was to assess the clinical implications of more prominent regurgitations, which also proved to be, very likely, physiological.

Improved ultrasound technology and technique have resulted in a marked increase in the ability to identify relatively subtle findings in the fetal heart, making it possible to characterize with greater nuance the anatomic and physiologic alterations throughout pregnancy. Trivial and mild TR that could not be detected before can now be easily identified. The prevalence of isolated TR varies with gestational age and technique of investigation. In the absence of any other systemic alterations, TR seems to be benign and represent changes in in-utero physiology that occur late in pregnancy.

It is important to point out some limitations of this study. Firstly, due to its retrospective design based on a pre-existing database, it was not possible to obtain demographic data of the 330 pregnant women. Therefore, a comprehensive characterization of our population could not be achieved. Albeit, according to the eligibility criteria, the pregnancies can be clearly defined as low-risk.

Another limitation of our study is that an echocardiographic evaluation was not available for all cases postnatally since it is not a current practice in our region to perform routinely an echocardiogram in otherwise healthy newborns. Since this is a retrospective study, we would not expect to have echocardiograms available for all included patients. However, we managed to obtain information from the majority of cases (62\%). This includes objective data from a questionnaire and echocardiography (available for only 14 cases). The aim of the postnatal evaluation was to confirm the antenatal prediction that the TR was a benign condition, i.e. of no clinical significance. Therefore, we considered adequate to state that a newborn that was discharged after being assessed by a pediatrician or neonatologist, and that was asymptomatic did not present a significant heart condition.

In conclusion, tricuspid valve regurgitation in fetuses with normal cardiac anatomy during the third trimester of pregnancy is a frequent condition in low-risk pregnancies and apparently is not associated with any cardiac abnormality or need for neonatal intervention.

\section{Author's contribution}

Lopes KRM conceived the presented idea, designed the study and performed the analysis, provided the data and wrote the paper with input from all authors. Cavalcante EVV, Melo NTL and Alves SA collected the data, contributed to the implementation of the 
research, discussed the results and commented on the manuscript. Alves JGB contributed to the study design and analysis, reviewed and made important contributions to the manuscript. Souza ASR contributed to the conception of the study, made important contributions to the analysis and interpretation of results, provided critical feedback and helped shape the manuscript.

\section{References}

1. Singh JP, Evans JC, Levy D, Larson MG, Freed LA, Fuller DL, Lehman B, Benjamin EJ. Prevalence and clinical determinants of mitral, tricuspid, and aortic regurgitation (The Framingham Heart Study). Am J Cardiol. 1999; 83: 897902.

2. Zhou J, Zhang Y, Gui Y, Chu C, Zhang C, Zhou Q, Zhang Y, Li X, Yan Y.Relationship Between Isolated Mild Tricuspid Valve Regurgitation in Second-Trimester Fetuses and Postnatal Congenital Cardiac Disorders. J Ultrasound Med. 2014; 33: 1677-82.

3. Respondek ML, Kammermeier M, Ludomirsky A, Weil SR, Huhta JC. The prevalence and clinical significance of fetal tricuspid valve regurgitation with normal heart anatomy. Am J Obstet Gynecol. 1994; 171: 1265-70.

4. Gembruch U, Smrcek JM. The prevalence and clinical significance of tricuspid valve regurgitation in normally grown fetuses and those with intrauterine growth retardation. Ultrasound Obstet Gynecol. 1997; 9: 374-82.

5. Harada K, Rice MJ, Shiota T, McDonald RW, Reller MD, Sahn DJ. Two-dimensional echocardiographic evaluation of ventricular systolic function in human fetuses with ductal constriction. Ultrasound Obstet Gynecol. 1997; 10 (4): 247 53.

6. McAuliffe FM, Trines J, Nield LE, Chitayat D, Jaeggi E, Hornberger LK. Early fetal echocardiography. Gynecol Obstet Invest. 2008; 65 (3): 162-8.

7. Scala C, Morlando M, Familiari A, Maggiore ULR, Ferrero S, D'Antonio F, Khalil A. Fetal Tricuspid Regurgitation in the First Trimester as a Screening Marker for Congenital Heart Defects: Systematic Review and Meta-Analysis. Fetal Diagn Ther.2017; 42: 1-8.
8. Kagan KO, Valencia C, Livanos P, Wright D, Nicolaides $\mathrm{KH}$. Tricuspid regurgitation in screening for trisomies 21 , 18 and 13 and Turner syndrome at $11+0$ to $13+6$ weeks of gestation. Ultrasound Obstet Gynecol. 2009; 33 (1): 18-22.

9. Pereira S, Ganapathy R, Syngelaki A, Maiz N, Nicolaides $\mathrm{KH}$. Contribution of fetal tricuspid regurgitation in firsttrimester screening for major cardiac defects. Obstet Gynecol. 2011; 117: 1384-91.

10. Messing B, Porat S, Imbar T, Valsky DV, Anteby EY, Yagel S. Mild tricuspid regurgitation: a benign fetal finding at various stages of pregnancy. Ultrasound Obstet Gynecol. 2005; 26: 606-10.

11. Anuwutnavin S, Wanitpongpan $\mathrm{P}$, Chanprapaph $\mathrm{P}$ Specificity of fetal tricuspid regurgitation in prediction of Down syndrome in Thai fetuses at 17-23 weeks of gestation. J Med Assoc Thai. 2009; 92: 1123-30.

12. Makikallio K, Rasanen J, Makikallio T, Vuolteenaho O, Huhta JC. Human fetal cardiovascular profile score and neonatal outcome in intrauterine growth restriction. Ultrasound Obstet Gynecol. 2008; 31: 48-54.

13. Tague L, Donofrio MT, Fulgium A, McCarter R, Limperopoulos C, Schidlow DN. Common findings in lategestation fetal echocardiography. J Ultrasound Med. 2017; 36 (12): 2431-7.

14. American Institute of Ultrasound in Medicine. AIUM practice guideline for the performance of fetal echocardiography. J Ultrasound Med. 2011; 30:127-36.

15. DeVore GR, Satou G, Sklansky M. 4D fetal echocardiography - An update. Echocardiography. 2017; 34 (12): 178898.
Received on July 23, 2020

Final version presented on December 15, 2020

Approved on February 24, 2021 